\title{
Baicalin inhibits growth and induces apoptosis of human osteosarcoma cells by suppressing the AKT pathway
}

\author{
YU LIU ${ }^{1}$, ZHENQIANG HONG ${ }^{1}$, PENG CHEN ${ }^{1}$, JINZHAO WANG ${ }^{1}$, YIMIN ZHOU ${ }^{1}$ and JIANYUN HUANG ${ }^{2}$ \\ ${ }^{1}$ Fujian University of Traditional Chinese Medicine, Key Laboratory of Orthopedics and Traumatology of \\ Traditional Chinese Medicine and Rehabilitation, Ministry of Education; ${ }^{2}$ Department of Orthopedics, \\ Third Affiliated Hospital of Fujian University of Traditional Chinese Medicine, Fuzhou, Fujian 350122, P.R. China
}

Received February 2, 2019; Accepted June 6, 2019

DOI: $10.3892 / \mathrm{ol} .2019 .10617$

\begin{abstract}
Osteosarcoma (OS) is one of the most prevalent types of bone malignancies with poor overall prognosis, and is reported mainly in children and adolescents. Therefore, the investigation of novel and efficient treatment strategies for patients with OS is required. Baicalin exhibits potential anticancer effects, including in OS. However, its therapeutic effect against OS and the underlying mechanisms have not been fully evaluated. In the present study, the effect of baicalin on the proliferation and apoptosis of OS cells and its underlying mechanism of AKT pathway activation was explored. Cell confluence and cell number counts revealed suppressed the growth of OS cells that were treated with baicalin. Analysis of cell viability, cell survival and cell cycle, as well as cell apoptosis revealed decreased cell viability and survival, induced cell cycle arrest and apoptosis of treated cells. Western blot analysis demonstrated significantly decreased ratios of phosphorylated-AKT/AKT and Bcl-2/Bax, and decreased protein levels of cyclin D1 and CDK4 in cells treated with baicalin. Thus, the findings from the present study suggest that the suppression of the AKT pathway may be the underlying mechanism of the antitumor effect of baicalin in OS cells.
\end{abstract}

\section{Introduction}

Osteosarcoma (OS), one of the most prevalent types of malignancies of the bone that predominantly affects children and adolescents, was the second leading cause of cancer-associated mortality in this group of individuals in the USA between 1973 and 2004 (1,2). Despite advances in therapeutic strategies (including chemotherapy and surgical resection), the 5-year

Correspondence to: Dr Jianyun Huang, Department of Orthopedics, Third Affiliated Hospital of Fujian University of Traditional Chinese Medicine, 363 Guobin Avenue, Fuzhou, Fujian 350122, P.R. China

E-mail: 444029074@qq.com

Key words: osteosarcoma, baicalin, proliferation, apoptosis, AKT pathway survival rate of patients with OS who are resistant to treatment or have metastases remains low (3). Moreover, due to poor overall prognosis, $>40 \%$ of patients develop recurrent or progressive disease following traditional first-line therapy (4). Due to the highly aggressive nature of these tumors, the poor therapeutic outcome and the development of chemoresistance, the exploration of novel and efficient treatment strategies for patients with OS is required (5).

Growing evidence indicates the potential of natural compounds as successful anticancer agents (6). Baicalin (baicalein 7-O- $\beta$-D-glucuronide), an important flavonoid, is found in the roots of the Chinese herb Huang Qin (Scutellaria baicalensis Georgi) (7). Baicalin exhibits a wide range of pharmacological properties, including against oxidation, tumor, inflammation and proliferation (8-11). Studies on the effect of this compound on various types of cancer cells indicated significantly suppressed tumor growth (12), induction of cell apoptosis and senescence (13-15), as well as inhibition of metastasis $(16,17)$. This is mediated by the suppression of multiple signaling pathways, including ERK, STAT3, $\beta$-catenin and p38 mitogen-activated protein kinase (MAPK) (16-19). Treatment of OS cells with baicalin significantly induces apoptosis and inhibits metastasis, through reactive oxygen species-mediated mitochondrial and TGF- $\beta$ pathways $(20,21)$. However, the role of baicalin in OS and its underlying mechanism has not been fully evaluated.

The AKT pathway, one of the most important intracellular signaling pathways, has been reported to involve a cascade of events that play an essential role in a variety of physiological and pathological processes, including proliferation, migration, cell growth and metabolism $(22,23)$. In most types of cancer in humans, the AKT pathway has been identified as one of the most important oncogenic pathways (24). In OS, the AKT pathway is frequently hyperactivated, playing a critical role in the initiation and development of OS, including tumorigenesis, proliferation, apoptosis and metastasis (25-28). Activation of the AKT pathway contributes to these processes in cancer by mediating the expression of its downstream genes, including cyclin D1, CDK4, anti-apoptotic protein Bcl-2 and pro-apoptotic protein Bax (29-32). Due to the essential role of the AKT pathway in OS, the inhibition of the AKT pathway has therapeutic potential in OS $(25,26,33-35)$ and natural compounds 
that target this pathway safely and effectively need to be further investigated.

In the present study, the role of baicalin on U-2 OS cell growth was assessed by cell confluence observation and performing cell number counts. The cell viability, cell survival, cell cycle and cell apoptosis of U-2 OS cells, following baicalin treatment, were further assessed. Moreover, the underlying mechanism of baicalin was explored by investigating the activation of the AKT pathway and its downstream effectors using western blotting.

\section{Materials and methods}

Antibodies and chemicals. McCoy's 5A medium, fetal bovine serum (FBS) and the cell cycle determination kit $\left[\mathrm{FxCycle}{ }^{\mathrm{TM}}\right.$ Propidium Iodide (PI)/RNase Staining solution; cat. no. F10797] were all purchased from Thermo Fisher Scientific, Inc. The mixture of penicillin and streptomycin was obtained from Hyclone; GE Healthcare Life Sciences. The Annexin V staining kit was provided by Nanjing KeyGen Biotech Co., Ltd. The Cell Counting kit-8 (CCK-8) was provided by Abbkine Scientific Co., Ltd. Baicalin and DMSO were obtained from Beijing Solarbio Science \& Technology Co., Ltd. The antibodies against GAPDH (cat. no. 5174), AKT (cat. no. 4691s), phosphorylated (p)-AKT (cat. no. 4060s), cyclin D1 (cat no. 2978s), CDK4 (cat. no. 12790), Bax (cat. no. 5023) and Bcl-2 (cat. no. 15071) were all purchased from Cell Signaling Technology, Inc.

Cell culture and baicalin treatment. U-2 OS cells were obtained from the Type Culture Collection of the Chinese Academy of Sciences. The cells were cultured in McCoy's $5 \mathrm{~A}$ medium, supplemented with $10 \%$ FBS and a mixture of $100 \mathrm{U} / \mathrm{ml}$ penicillin and $100 \mathrm{mg} / \mathrm{ml}$ streptomycin, in a humidified atmosphere of $37^{\circ} \mathrm{C}$ and $5 \% \mathrm{CO}_{2}$. The cells were cultured to $80-90 \%$ confluence and were not used after $>20$ passages. The cells were seeded in multiple plates and treated with various concentrations of baicalin $(25,50$ or $100 \mu \mathrm{M}$; dissolved in DMSO). The concentrations were selected based on a preliminary study (data not shown). Equal volume of DMSO $(\leq 0.5 \%)$ was added to wells as a control treatment.

Cell confluence observation. U-2 OS cells $\left(0.4 \times 10^{5}\right.$ cells/well) were seeded on a 6 -well plate for $24 \mathrm{~h}$, followed by treatment with $0,25,50$ or $100 \mu \mathrm{M}$ baicalin for $24 \mathrm{~h}$. The confluence of cells was observed and images of each well were captured using a phase-contrast inverted light microscope (Leica Microsystems $\mathrm{GmbH}$ ) at a magnification of x100.

Cell number counts. Following the observation of cell confluence, the cells were trypsinized and diluted with fresh medium. An equal volume of medium containing cells was mixed with $0.2 \%$ trypan blue solution (Sigma Aldrich; Merck KGaA) and the cell number was counted using the Countstar Automated Cell Counter (ALIT Life Science Co., Ltd.).

CCK-8 assay. U-2 OS cells ( $2 \times 10^{3}$ cells/well) were seeded on a 96-well plate for $24 \mathrm{~h}$ and then treated with various concentrations of baicalin $(0,25,50$ or $100 \mu \mathrm{M})$. Following treatment for 24, 48 and $72 \mathrm{~h}, 10 \mu \mathrm{l} \mathrm{CCK}-8$ reagent (Abbkine Scientific Co., Ltd.) was added to each well and the cells were incubated at $37^{\circ} \mathrm{C}$ for an additional $2 \mathrm{~h}$. The absorbance was measured at $450 \mathrm{~nm}$ using an Infinite 200 Pro microplate reader (Tecan Group, Ltd.).

Cell colony-formation analysis. Following the cell number counts for each treatment group, U-2 OS cells treated with or without baicalin were seeded on 12-well plates ( 500 cells/well), and the medium was changed every 2-3 days. After culture for 8-10 days, the colonies formed were washed with PBS twice and fixed with 4\% paraformaldehyde for $15 \mathrm{~min}$ at room temperature (RT), followed by $0.01 \%$ crystal violet staining for $15 \mathrm{~min}$ at RT. Images were captured using an electronic camera (cat. no. DS126201; Canon, Inc.) and the numbers of colonies were recorded. The number of colonies formed was normalized to the control cells.

Cell cycle analysis. U-2 OS cells $\left(0.4 \times 10^{5}\right.$ cells/well) were seeded on a 6 -well plate, incubated for $24 \mathrm{~h}$, and treated with $0,25,50$ or $100 \mu \mathrm{M}$ baicalin for $24 \mathrm{~h}$. The cells were harvested and fixed with $70 \%$ ethanol at $4^{\circ} \mathrm{C}$ overnight. The fixed cells were washed twice with cold PBS and incubated for $30 \mathrm{~min}$ with PI/RNase at RT. The fluorescence signal was detected by the FL2 channel of a flow cytometer and the proportion of DNA at each phase was analyzed using ModFit LT software version 3.0 (Verity Software House, Inc.).

Annexin V staining and cell apoptosis analysis. U-2 OS cells $\left(0.4 \times 10^{5}\right.$ cells $/$ well $)$ were seeded on a 6 -well plate and incubated for $24 \mathrm{~h}$, after treatment with $0,25,50$ or $100 \mu \mathrm{M}$ baicalin for $24 \mathrm{~h}$. The cells were harvested with trypsin without EDTA and washed twice with PBS. The collected cells were incubated with Annexin V-phycoerythrin solution for $15 \mathrm{~min}$ at RT. The cells were sorted using a flow cytometer (FACSCalibur ${ }^{\mathrm{TM}}$; Becton Dickinson Company). The percentage of cells at the early stage of apoptosis was calculated according to Annexin V-positivity using Cell Quest Pro software (version 6.0; Becton, Dickinson and Company).

Western blotting. U-2 OS cells were cultured in $25-\mathrm{cm}^{2}$ flasks at a density of $1.5 \times 10^{6}$ cells/flask in $5 \mathrm{ml}$ medium for $24 \mathrm{~h}$, and then treated with $0,25,50$ or $100 \mu \mathrm{M}$ baicalin for $24 \mathrm{~h}$. After harvesting, the cells were lysed with ice-cold RIPA lysis buffer (Beyotime Institute of Biotechnology), containing protease and phosphatase inhibitor cocktails (Roche Diagnostics) for $30 \mathrm{~min}$, and centrifuged at $18,894 \mathrm{xg}$ at $4^{\circ} \mathrm{C}$ for 10 min to remove sediment. The concentration of soluble protein was determined by BCA assay (Thermo Fisher Scientific, Inc.). Protein samples $(50 \mu \mathrm{g})$ were separated by $10 \%$ SDS-PAGE and transferred to PVDF membranes (EMD Millipore). The PVDF membranes were blocked with $5 \%$ skimmed milk at RT for $1 \mathrm{~h}$. The membranes were then incubated with primary antibodies (1:1,000 dilution) against phosphorylated (p-)AKT, AKT, cyclin D1, CDK4, Bax and $\mathrm{Bcl}-2$ overnight at $4^{\circ} \mathrm{C}$. The blots were then probed with a goat anti-rabbit horseradish peroxidase-conjugated secondary antibody (1:2,000 dilution) at RT for $2 \mathrm{~h}$, followed by detection using enhanced chemiluminescence (Thermo Fisher Scientific, Inc.) with the ChemiDoc XRS+ imaging system (Bio-Rad Laboratories, Inc.). The intensities of bands were quantified relative to the intensity of GAPDH bands using the ImageJ software (version 1.46; National Institutes of Health). The levels of target proteins were expressed relative to the levels in untreated cells, defined as 1.00. 
A

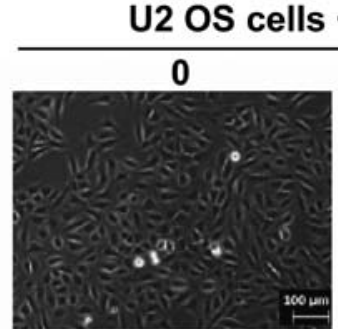

50

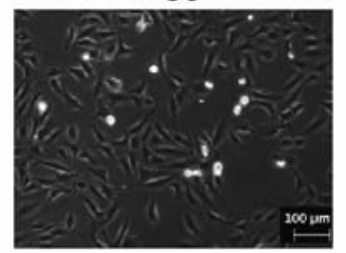

B

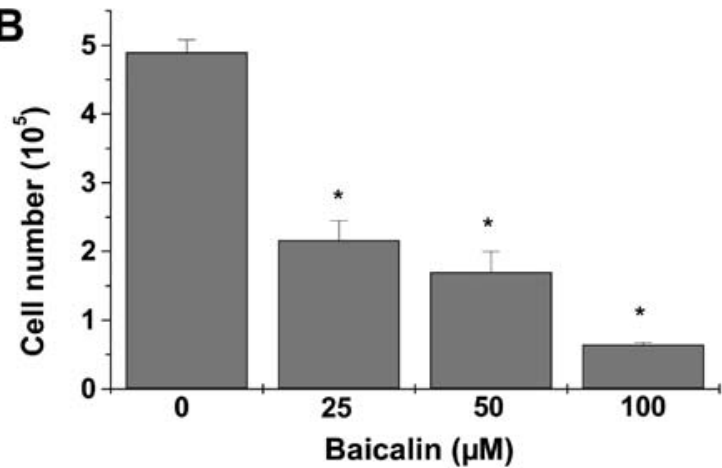

Figure 1. Effect of baicalin treatment on the growth of U-2 OS cells. Cells were treated with $0,25,50$ or $100 \mu \mathrm{M}$ baicalin for $24 \mathrm{~h}$. (A) Cell confluence was observed and images were captured using a phase-contrast inverted microscope. (B) Cells were counted using the Countstar Automated Cell Counter, using the trypan blue exclusion principle. Magnification, x 100 . Data are presented as mean \pm SD of $n=6$ of three independent experiments. ${ }^{*} \mathrm{P}<0.05$ vs. untreated $\mathrm{U}-2$ OS cells.

Statistical analysis. All statistical analyses were conducted using the SPSS version 20.0 statistical software (IBM Corp.). The data are presented as the mean \pm standard deviation of three independent experiments. The significance of the differences between the groups ( $>3$ groups) was tested using ANOVA, followed by the Least Significant Difference test. $\mathrm{P}<0.05$ was considered to indicate a statistically significant difference.

\section{Results}

Baicalin suppresses the growth of $U-2$ OS cells. Cell confluence observation by microscopy revealed decreased cell confluence of cultured U-2 OS cells that were treated at various concentrations of baicalin (25, 50 or $100 \mu \mathrm{M}$; Fig. 1A). Moreover, cell number counts, using trypan blue staining, demonstrated significantly decreased number of live cells following treatment with $25-100 \mu \mathrm{M}$ baicalin $(\mathrm{P}<0.05$ vs. untreated U-2 OS cells; Fig. 1B). Thus, baicalin suppresses the growth of U-2 OS cells.

Baicalin decreases cell viability and cell survival of $U-2$ OS cells. The cell viability of U-2 OS cells was determined using the CCK- 8 assay. This demonstrated markedly decreased cell
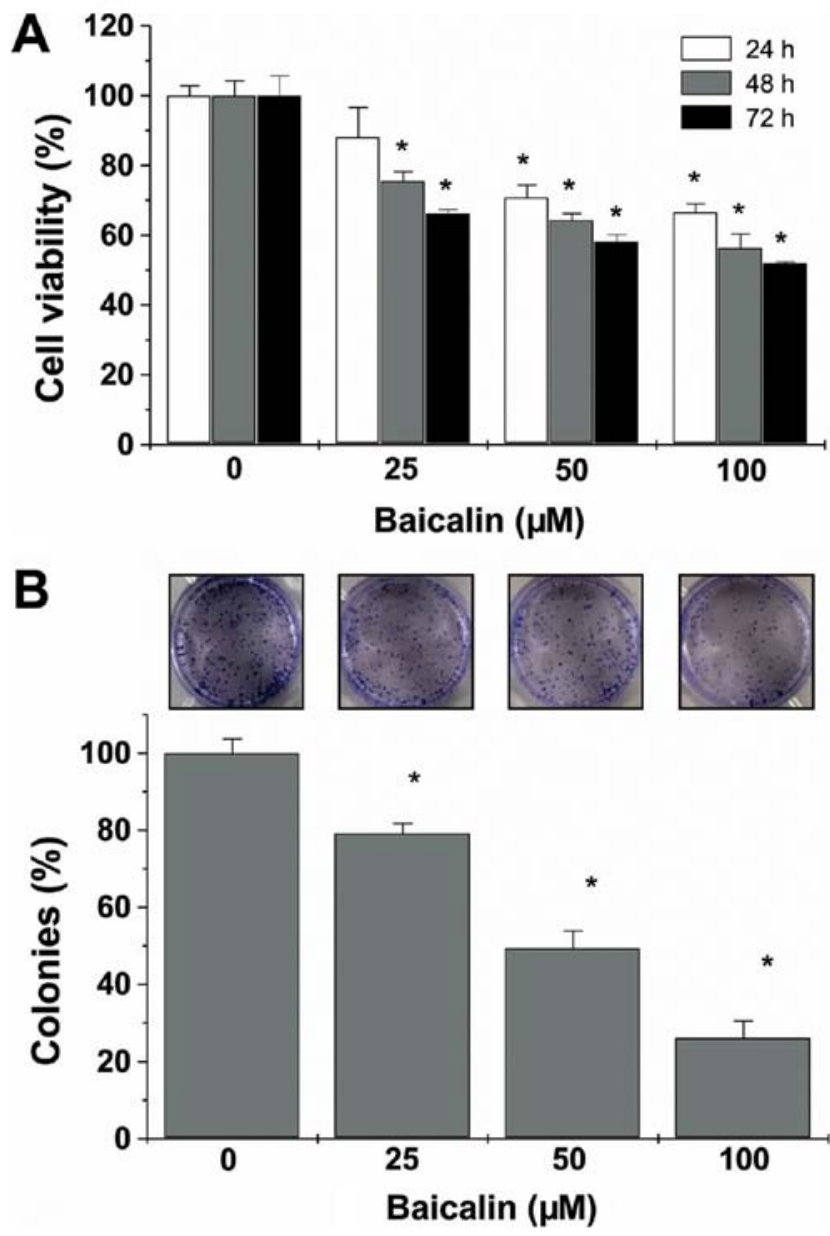

Figure 2. Effect of baicalin treatment on viability and survival of U-2 OS cells. (A) U-2 OS cells were treated with $0,25,50$ or $100 \mu \mathrm{M}$ baicalin for 24 , 48 or 72 h. Cell Counting Kit- 8 was used to measure cell viability relative to the untreated U-2 OS cells. (B) U-2 OS cells were treated with $0,25,50$ or $100 \mu \mathrm{M}$ baicalin for $24 \mathrm{~h}$ and reseeded in 12-well plates to analyze colony formation. Representative images are shown and the data are normalized to the untreated U-2 OS cells. Data are presented as mean \pm SD of three independent experiments. $\mathrm{P}<0.05$ vs. untreated U-2 OS cells.

viability of U-2 OS cells that were treated with baicalin $(25,50$ or $100 \mu \mathrm{M})$ at various time points $(24,48$ or $72 \mathrm{~h})$ compared with untreated cells (all $\mathrm{P}<0.05$, except $25 \mu \mathrm{M}$ baicalin treatment for $24 \mathrm{~h}$; Fig. 2A). The images and calculations of the colonies revealed significantly decreased numbers of colonies at the various concentrations of baicalin (all $\mathrm{P}<0.05$ vs. untreated cells; Fig. 2B).

Baicalin induces the entry of U-2 OS cells into the $G_{0} / G_{I}$ phase. In order to explore the underlying mechanism of the effect of baicalin on cell cycle progression, cell cycle analysis was conducted (Fig. 3). PI staining followed by FACS analysis indicated a significantly increased percentage of U-2 OS cells at the $\mathrm{G}_{0} / \mathrm{G}_{1}$ phase after $50-\mu \mathrm{M}(36.55 \pm 1.16 \%)$ and $100-\mu \mathrm{M}$ $(36.37 \pm 1.77 \%)$ baicalin treatment compared with untreated U-2 OS cells $(31.39 \pm 1.84 \%)$ (both $\mathrm{P}<0.05)$. The percentage of $\mathrm{U}-2$ OS cells at the $\mathrm{S}$ phase was significantly decreased following treatment with $25(42.79 \pm 1.18 \%), 50(43.20 \pm 0.42 \%)$ and $100 \mu \mathrm{M}$ (38.24 \pm 1.43$)$ baicalin compared with untreated U-2 OS cells $(48.03 \pm 0.32 \%)($ all $\mathrm{P}<0.05)$. In addition, the percentage of cells at the $\mathrm{G}_{2} / \mathrm{M}$ phase was increased following treatment with 25 
U2 OS cells + Baicalin $(\mu \mathrm{M})$
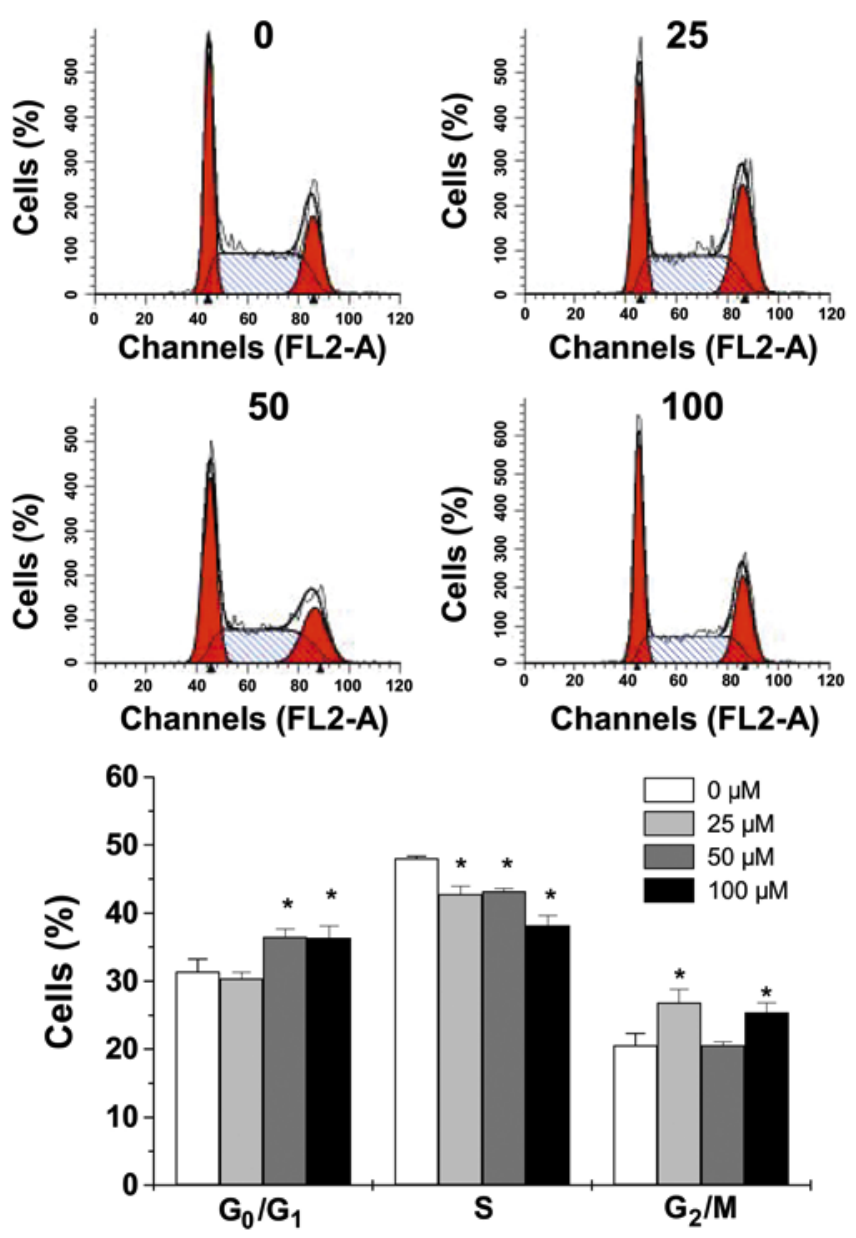

Figure 3. Effect of baicalin treatment on cell cycle progression in U-2 OS cells. U-2 OS cells were treated with $0,25,50$ or $100 \mu \mathrm{M}$ baicalin for $24 \mathrm{~h}$. The treated cells were stained with propidium iodide solution in order to analyze cell cycle distribution with flow cytometry. The percentage of cells in the $G_{0} / G_{1}, S$ and $G_{2} / M$ phases was measured. The data are represented as mean \pm SD of three independent experiments. ${ }^{*} \mathrm{P}<0.05$ vs. untreated U-2 OS cells; FL2-A, fluorescent light-area.

and $100 \mu \mathrm{M}$ baicalin $(\mathrm{P}<0.05)$, whereas $50 \mu \mathrm{M}$ baicalin treatment had no effect.

Baicalin induces apoptosis of U-2 OS cells. Annexin V staining followed by FACS analysis revealed a significant shift in the peak of fluorescence to the right and significantly increased percentage of U-2 OS cells that were positively stained with Annexin V following treatment with 25, 50 and $100 \mu \mathrm{M}$ baicalin $(9.03 \pm 1.90,13.28 \pm 1.44$ and $37.00 \pm 1.42 \%$, respectively), compared with untreated cells (all $\mathrm{P}<0.05$; Fig. 4).

Baicalin treatment suppresses the activation of the AKT pathway. The underlying mechanism of the antitumor effect of baicalin was further investigated by western blot analysis (Fig. 5A). As shown in Fig. 5B, treatment with baicalin significantly decreased the levels of $\mathrm{p}$-AKT relative to total AKT (all $\mathrm{P}<0.05$ vs. untreated cells). Determination of the protein levels of its downstream effectors revealed significantly down- regulated expression of CDK4 at all tested concentrations, and cyclin D1 at $100 \mu \mathrm{M}$ only, and decreased Bcl-2/Bax ratio in $\mathrm{U}-2$ OS cells treated with baicalin (all $\mathrm{P}<0.05$ vs. untreated cells; Fig. 5C-E).

\section{Discussion}

Treatment of OS by chemotherapy and surgical resection is limited, and patients who are resistant to treatment or have metastasis have poor overall survival (3). Thus, the development of effective therapeutic approaches for OS, with low toxicity and minimal side effects, is required. A number of natural compounds, including kaempferol and oleanolic acid, exhibit potential as anticancer agents with low toxicity $(36,37)$. Baicalin is a naturally bioactive compound extracted from the Chinese herb Huang Qin (Scutellaria baicalensis Georgi) (7). Previous studies demonstrated significant effects of baicalin, such as those against oxidation, tumor, inflammation and proliferation (8-11). Moreover, treatment of various cancer cells with baicalin significantly suppresses tumor growth and metastasis, via multiple downstream signaling pathways (13-19). Other studies on OS revealed significant induction of cell apoptosis and inhibition of metastasis following baicalin treatment $(20,21)$. In the present study, the role of baicalin on the growth of OS cells was further explored. This revealed significantly decreased cell confluence and number of OS cells. Moreover, the CCK-8 and colony-formation assays revealed significantly decreased cell viability of OS cells treated with baicalin, suggesting the potential of baicalin as an anticancer therapy for OS. However, the effect of baicalin treatment on tumor growth, metastasis and chemotherapy resistance in OS should be further assessed in vivo.

As with most cancer cells, OS cells are characterized by imbalanced cell proliferation and apoptosis. Therefore, the present study determined the progression of the cell cycle in OS cells and found that treatment with baicalin significantly decreased the percentage of cells at the $\mathrm{S}$ phase, whereas as an increased percentage at the $\mathrm{G}_{0} / \mathrm{G}_{1}$ phase was observed. Cyclin D1, together with specific kinases (CDK4 and CDK6), plays an essential role in the regulation of the cell cycle at the $\mathrm{G}_{0} / \mathrm{G}_{1}$ restriction points. Therefore, western blot analysis of cyclin D1 and CDK4 was performed on U-2 OS cells treated with baicalin, revealing their downregulation. However, other regulators (including p21, p27 and CDK6) that are involved in the control of cell cycle checkpoints at the $G_{0} / G_{1}$ and $S$ phases should be further evaluated in future studies. Moreover, the percentage of cells at the $\mathrm{G}_{2} / \mathrm{M}$ phase was increased following treatment with 25 and $100 \mu \mathrm{M}$ baicalin, whereas $50 \mu \mathrm{M}$ baicalin had no effect. This observation can be further explored in future studies.

Evading apoptosis is another hallmark of cancer cells, which is mediated by anti-proliferative proteins (such as Bcl-2) and anti-apoptotic proteins (such as Bax). Analysis of the fragmented DNA (a characteristic of late apoptosis) by PI staining revealed a low percentage of cells at the Sub-G1 phase. This was in agreement with the finding of an increased percentage of apoptotic cells by Annexin V staining. However, the role of baicalin in the apoptotic status of cells should be further investigated using double staining of Annexin V-allophycocyanin 

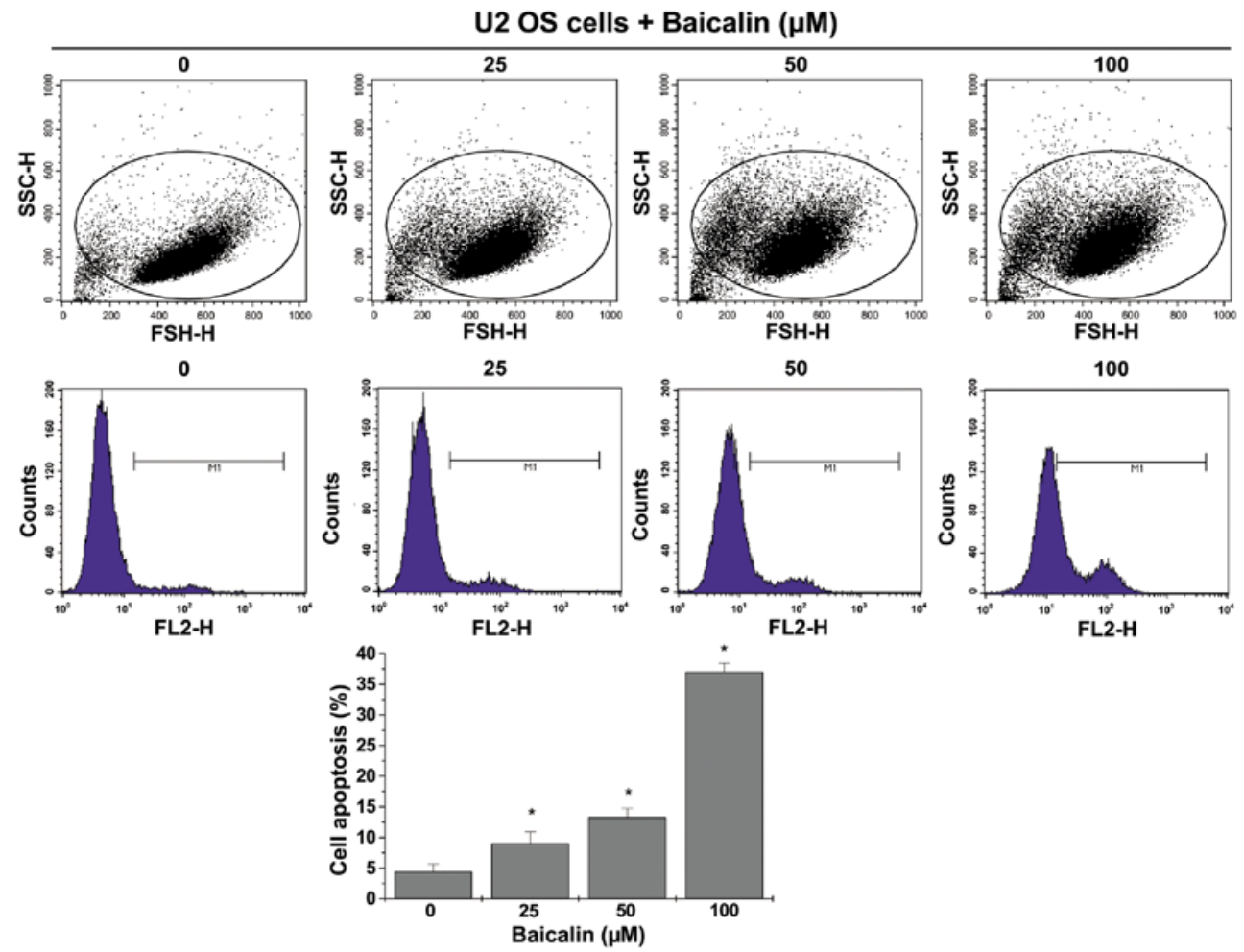

Figure 4. Effect of baicalin treatment on apoptosis of U-2 OS cells. Cells were treated with $0,25,50$ or $100 \mu \mathrm{M}$ baicalin for $24 \mathrm{~h}$. The percentage of apoptotic cells was measured using Annexin V-phycoerythrin staining analyzed with flow cytometry. The data are presented as mean \pm SD of three independent experiments. "P<0.05 vs. untreated U-2 OS cells. FSH-H, forward angular scattering-height; SSC-H, side scatter-height; FL2-H, fluorescent light-height.
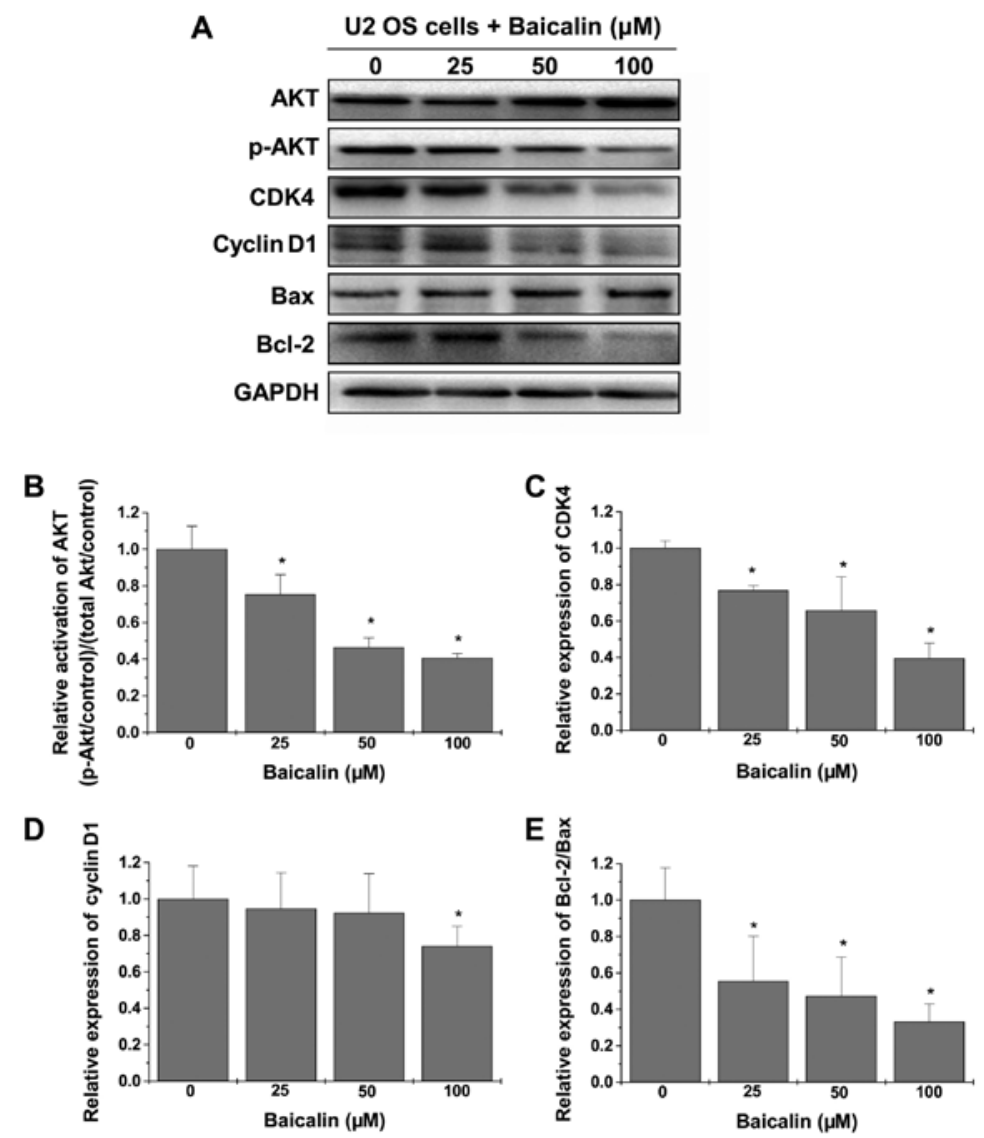

Figure 5. Effect of baicalin treatment on the AKT pathway. U-2 OS cells were treated with 0, 25, 50 or $100 \mu \mathrm{M}$ baicalin for $24 \mathrm{~h}$. (A) The protein expression of p-AKT, AKT, cyclin D1, CDK4, Bax and Bcl-2 was determined by western blot analysis. GAPDH was used as the internal control. (B-E) The intensities of bands were normalized against those of GAPDH and quantified relative to those of the untreated control cells. The data are presented as mean \pm SD of three independent experiments. ${ }^{*} \mathrm{P}<0.05$ vs. untreated U-2 OS cells. p-AKT, phosphorylated AKT. 
with PI or 7-aminoactinomycin D. Furthermore, western blot analysis revealed enhanced expression of the anti-apoptotic protein Bax, whereas expression of the anti-apoptotic protein Bcl-2 was decreased, in U-2 OS cells treated with baicalin. These findings thus suggest induced cell cycle arrest and cell apoptosis via the downregulation of cell cycle regulators cyclin D1 and CDK4, and anti-apoptotic protein Bcl-2. However, the underlying mechanism of baicalin needs to be further investigated with omics technologies, including cDNA arrays and sequencing.

Multiple signaling pathways (including ERK, p38 MAPK and STAT3) have been reported to play a critical role in the development and prognosis of OS, which has been reported to be suppressed by baicalin, leading to the inhibition of tumor growth and metastasis (12-19). However, to the best of our knowledge, the regulatory effect of baicalin on the AKT pathway has never been evaluated in OS. In OS, hyperactivation of the AKT pathway is critical in tumorigenesis, proliferation, cell cycle, apoptosis and metastasis, by regulating the expression of its downstream effectors (such as cyclin D1, CDK4, Bcl-2 and Bax) (25-32). Thus, the downregulation of cyclin D1, CDK4, Bcl-2 expression and upregulation of Bax expression observed in OS cells after baicalin treatment prompts further exploration of the role of this compound on the activation of the AKT pathway.

In conclusion, baicalin significantly suppressed the growth of OS cells, inhibited cell viability and survival, and induced cell cycle arrest and apoptosis. Mechanistic studies revealed suppression of the AKT pathway and decreased protein expression of cyclin D1, CDK4 and Bcl-2/Bax ratio as the possible mechanism of the baicalin antitumor effect in OS. The present study provides a basis to further explore the treatment of OS with baicalin in the future, using in vitro and in vivo studies.

\section{Acknowledgements}

Not applicable.

\section{Funding}

This study was supported by the National Nature Science Foundation of China (grant no. 81373659).

\section{Availability of data and materials}

The datasets generated and/or analyzed during the current study are available from the corresponding author on reasonable request.

\section{Authors' contributions}

YL and JH conceived and designed the experiments. YL, PC and JW performed the experiments on cells. $\mathrm{ZH}$ and $\mathrm{YZ}$ conducted the western blot analysis. YL and JW conducted the data analysis. YL and PC produced and revised the manuscript. All authors read and approved the final manuscript.

\section{Ethics approval and consent to participate}

Not applicable.

\section{Patient consent for publication}

Not applicable.

\section{Competing interests}

The authors declare that they have no competing interests.

\section{References}

1. Mirabello L, Troisi RJ and Savage SA: Osteosarcoma incidence and survival rates from 1973 to 2004: Data from the surveillance, epidemiology, and end results program. Cancer 115: 1531-1543, 2009.

2. Dong F, Liu T, Jin H and Wang W: Chimaphilin inhibits human osteosarcoma cell invasion and metastasis through suppressing the TGF- $\beta 1$-induced epithelial-to-mesenchymal transition markers via PI-3K/Akt, ERK1/2, and Smad signaling pathways. Can J Physiol Pharmacol 96: 1-7, 2018.

3. Wang Y, Li L, Shao N, Hu Z, Chen H, Xu L, Wang C, Cheng Y and Xiao J: Triazine-modified dendrimer for efficient TRAIL gene therapy in osteosarcoma. Acta Biomater 17: 115-124, 2015.

4. Bienemann K, Staege MS, Howe SJ, Sena-Esteves M, Hanenberg H and Kramm CM: Targeted expression of human folylpolyglutamate synthase for selective enhancement of methotrexate chemotherapy in osteosarcoma cells. Cancer Gene Ther 20: 514-520, 2013.

5. Wu G, Liang Q and Liu Y: Primary osteosarcoma of frontal bone: A case report and review of literature. Medicine (Baltimore) 96: e9392, 2017.

6. Liu J, Zhang L, Ren Y, Gao Y, Kang L and Qiao Q: Anticancer and immunoregulatory activity of Gynostemma pentaphyllum polysaccharides in H22 tumor-bearing mice. Int J Biol Macromol 69: 1-4, 2014.

7. Li B, Wan L, Li Y, Yu Q, Chen P, Gan R, Yang Q, Han Y and Guo C: Baicalin, a component of Scutellaria baicalensis, alleviates anorexia and inhibits skeletal muscle atrophy in experimental cancer cachexia. Tumour Biol 35: 12415-12425, 2014

8. Gao Z, Huang K and Xu H: Protective effects of flavonoids in the roots of Scutellaria baicalensis Georgi against hydrogen peroxide-induced oxidative stress in HS-SY5Y cells. Pharmacol Res 43: 173-178, 2001.

9. Ikezoe T, Chen, SS, Heber D, Taguchi $\mathrm{H}$ and Koeffler HP: Baicalin is a major component of PC-SPES which inhibits the proliferation of human cancer cells via apoptosis and cell cycle arrest. Prostate 49: 285-292, 2001.

10. Shen YC, Chiou WF, Chou YC and Chen CF: Mechanisms in mediating the anti-inflammatory effects of baicalin and baicalein in human leukocytes. Eur J Pharmacol 465: 171-181, 2003.

11. Dong LH, Wen JK, Miao SB, JiaZ,Hu HJ, Sun RH,Wu Y and Han M: Baicalin inhibits PDGF-BB-stimulated vascular smooth muscle cell proliferation through suppressing PDGFR $\beta$-ERK signaling and increase in p27 accumulation and prevents injury-induced neointimal hyperplasia. Cell Res 20: 1252-1262, 2010.

12. Chen WC, Kuo TH, Tzeng YS and Tsai YC: Baicalin induces apoptosis in SW620 human colorectal carcinoma cells in vitro and suppresses tumor growth in vivo. Molecules 17: 3844-3857, 2012.

13. Yu Y, Pei M and Li L: Baicalin induces apoptosis in hepatic cancer cells in vitro and suppresses tumor growth in vivo. Int J Clin Exp Med 8: 8958-8967, 2015.

14. Wang H, Li H, Chen F, Luo J, Gu J, Wang H, Wu H and $\mathrm{Xu}$ Y: Baicalin extracted from Huangqin (Radix Scutellariae Baicalensis) induces apoptosis in gastric cancer cells by regulating $\mathrm{B}$ cell lymphoma (Bcl-2)/Bcl-2-associated $\mathrm{X}$ protein and activating caspase-3 and caspase-9. J Tradit Chin Med 37: 229-235, 2017.

15. Dou J, Wang Z, Ma L, Peng B, Mao K, Li C, Su M, Zhou C and Peng G: Baicalein and baicalin inhibit colon cancer using two distinct fashions of apoptosis and senescence. Oncotarget 9: 20089-20102, 2018.

16. Zhou T, Zhang A, Kuang G, Gong X, Jiang R, Lin D, Li J, Li H, Zhang X, Wan J and Li H: Baicalin inhibits the metastasis of highly aggressive breast cancer cells by reversing epithelial-to-mesenchymal transition by targeting $\beta$-catenin signaling. Oncol Rep 38: 3599-3607, 2017. 
17. Wang XF, Zhou QM, Du J, Zhang H, Lu YY and Su SB: Baicalin suppresses migration, invasion and metastasis of breast cancer via p38MAPK signaling pathway. Anticancer Agents Med Chem 13: 923-931, 2013

18. Wang Z, Ma L, Su M, Zhou Y, Mao K, Li C, Peng G, Zhou C, Shen B and Dou J: Baicalin induces cellular senescence in human colon cancer cells via upregulation of DEPP and the activation of Ras/Raf/MEK/ERK signaling. Cell Death Dis 9: 217, 2018.

19. Wang Q, Xu H and Zhao X: Baicalin inhibits human cervical cancer cells by suppressing protein kinase $\mathrm{C} /$ signal transducer and activator of transcription (PKC/STAT3) signaling pathway. Med Sci Monit 24: 1955-1961, 2018.

20. Wan D and Ouyang H: Baicalin induces apoptosis in human osteosarcoma cell through ROS-mediated mitochondrial pathway. Nat Prod Res 32: 1996-2000, 2018.

21. Wang Y, Wang H, Zhou R, Zhong W, Lu S, Ma Z and Chai Y: Baicalin inhibits human osteosarcoma cells invasion, metastasis, and anoikis resistance by suppressing the transforming growth factor- $\beta 1$-induced epithelial-to-mesenchymal transition. Anticancer Drugs 28: 581-587, 2017.

22. Abeyrathna P and Su Y: The critical role of Akt in cardiovascular function. Vascul Pharmacol 74: 38-48, 2015.

23. Martini M, De Santis MC, Braccini L, Gulluni F and Hirsch E PI3K/AKT signaling pathway and cancer: An updated review. Ann Med 46: 372-383, 2014.

24. Porta C, Paglino C and Mosca A: Targeting PI3K/Akt/mTOR signaling in cancer. Front Oncol 4: 64, 2014.

25. Zhang J, Yu XH, Yan YG, Wang C and Wang WJ: PI3K/Akt signaling in osteosarcoma. Clin Chim Acta 444: 182-192, 2015 .

26. Zhou Y, Zhu LB, Peng AF, Wang TF, Long XH, Gao S, Zhou RP and Liu ZL: LY294002 inhibits the malignant phenotype of osteosarcoma cells by modulating the phosphatidylinositol 3-kinase/Akt/fatty acid synthase signaling pathway in vitro. Mol Med Rep 11: 1352-1357, 2015.

27. Zhang B, Li YL, Zhao JL, Zhen O, Yu C, Yang BH and Yu XR: Hypoxia-inducible factor-1 promotes cancer progression through activating AKT/Cyclin D1 signaling pathway in osteosarcoma. Biomed Pharmacother 105: 1-9, 2018

28. Ren L, Hong ES, Mendoza A, Issaq S, Tran Hoang C, LeBlanc A and Khanna C: Metabolomics uncovers a link between inositol metabolism and osteosarcoma metastasis. Oncotarget 8 : 38541-38553, 2017.
29. Zhang Y, Hu H, Song L, Cai L, Wei R and Jin W: Epirubicin-mediated expression of $\mathrm{miR}-302 \mathrm{~b}$ is involved in osteosarcoma apoptosis and cell cycle regulation. Toxicol Lett 222: 1-9, 2013.

30. Zhang K, Tian F, Zhang Y, Zhu Q, Xue N, Zhu H, Wang H and Guo X: MACC1 is involved in the regulation of proliferation, colony formation, invasion ability, cell cycle distribution, apoptosis and tumorigenicity by altering Akt signaling pathway in human osteosarcoma. Tumour Biol 35: 2537-2548, 2014.

31. Li G, Cai M, Fu D, Chen K, Sun M, Cai Z and Cheng B: Heat shock protein $90 \mathrm{~B} 1$ plays an oncogenic role and is a target of microRNA-223 in human osteosarcoma. Cell Physiol Biochem 30: 1481-1490, 2012.

32. AtifF, Yousuf S and Stein DG: Anti-tumor effects of progesterone in human glioblastoma multiforme: Role of PI3K/Akt/mTOR signaling. J Steroid Biochem Mol Biol 146: 62-73, 2015.

33. Zhuo B, Li Y, Li Z, Qin H, Sun Q, Zhang F, Shen Y, Shi Y and Wang R: PI3K/Akt signaling mediated Hexokinase-2 expression inhibits cell apoptosis and promotes tumor growth in pediatric osteosarcoma. Biochem Biophys Res Commun 464: 401-406, 2015.

34. Wang Y, Wan D, Zhou R, Zhong W, Lu S and Chai Y: Geraniin inhibits migration and invasion of human osteosarcoma cancer cells through regulation of PI3K/Akt and ERK1/2 signaling pathways. Anticancer Drugs 28: 959-966, 2017.

35. Angulo P, Kaushik G, Subramaniam D, Dandawate P, Neville K, Chastain K and Anant S: Natural compounds targeting major cell signaling pathways: A novel paradigm for osteosarcoma therapy. J Hematol Oncol 10: 10, 2017.

36. Lee J and Kim JH: Kaempferol inhibits pancreatic cancer cell growth and migration through the blockade of EGFR-related pathway in vitro. PLoS One 11: e0155264, 2016.

37. Li L, Wei L, Shen A, Chu J, Lin J and Peng J: Oleanolic acid modulates multiple intracellular targets to inhibit colorectal cancer growth. Int J Oncol 47: 2247-2254, 2015.

This work is licensed under a Creative Commons Attribution-NonCommercial-NoDerivatives 4.0 International (CC BY-NC-ND 4.0) License. 